\title{
Renewable Energy Provisioning for ICT Services in a Future Internet
}

\author{
Kim Khoa Nguyen ${ }^{1}$, Mohamed Cheriet ${ }^{1}$, Mathieu Lemay ${ }^{2}$, Bill St. Arnaud ${ }^{3}$, \\ Victor Reijs ${ }^{4}$, Andrew Mackarel ${ }^{4}$, Pau Minoves ${ }^{5}$, Alin Pastrama ${ }^{6}$, and \\ Ward Van Heddeghem ${ }^{7}$ \\ ${ }^{1}$ Ecole de Technologie Superieure, University of Quebec, Canada \\ kim.nguyen@synchromedia.ca, Mohamed.Cheriet@et.smtl.ca \\ ${ }^{2}$ Inocybe Technologies, Canada \\ mlemay@inocybe.ca \\ ${ }^{3}$ St. Arnaud Walker \& Associates, Canada \\ bill.st.arnaudegmail.com \\ ${ }^{4}$ HEAnet, Ireland \\ \{victor.reijs, andrew.mackarel\}@heanet.ie \\ 5 i2CAT Foundation, Spain \\ pau.minoves@i2cat.net \\ ${ }^{6}$ Nordunet, Iceland \\ alin@nordu.net \\ ${ }^{7}$ Interdisciplinary institute for BroadBand Technology, Belgium. \\ ward.vanheddeghemaintec.ugent. be
}

\begin{abstract}
As one of the first worldwide initiatives provisioning ICT (Information and Communication Technologies) services entirely based on renewable energy such as solar, wind and hydroelectricity across Canada and around the world, the GreenStar Network (GSN) is developed to dynamically transport user services to be processed in data centers built in proximity to green energy sources, reducing GHG (Greenhouse Gas) emissions of ICT equipments. Regarding the current approach, which focuses mainly in reducing energy consumption at the micro-level through energy efficiency improvements, the overall energy consumption will eventually increase due to the growing demand from new services and users, resulting in an increase in GHG emissions. Based on the cooperation between Mantychore FP7 and the GSN, our approach is, therefore, much broader and more appropriate because it focuses on GHG emission reductions at the macro-level. Whilst energy efficiency techniques are still encouraged at low-end client equipments, the heaviest computing services are dedicated to virtual data centers powered completely by green energy from a large abundant reserve of natural resources, particularly in northern countries.
\end{abstract}

Keywords: Green Star Network, Mantychore FP7, green ICT, Future Internet

\section{Introduction}

Nowadays, reducing greenhouse gas (GHG) emissions is becoming one of the most challenging research topics in Information and Communication Technologies (ICT) 
because of the alarming growth of indirect GHG emissions resulting from the overwhelming utilization of ICT electrical devices [1]. The current approach when dealing with the ICT GHG problem is improving energy efficiency, aimed at reducing energy consumption at the micro level. Research projects following this direction have focused on micro-processor design, computer design, power-on-demand architectures and virtual machine consolidation techniques. However, a micro-level energy efficiency approach will likely lead to an overall increase in energy consumption due to the Khazzoom-Brookes postulate (also known as Jevons paradox) [2], which states that "energy efficiency improvements that, on the broadest considerations, are economically justified at the micro level, lead to higher levels of energy consumption at the macro level". Therefore, we believe that reducing GHG emissions at the macro level is a more appropriate solution. Large ICT companies, like Microsoft which consumes up to $27 \mathrm{MW}$ of energy at any given time [1], have built their data centers near green power sources. Unfortunately, many computing centers are not so close to green energy sources. Thus, green energy distributed network is an emerging technology, given that losses incurred in energy transmission over power utility infrastructures are much higher than those caused by data transmission, which makes relocating a data center near a renewable energy source a more efficient solution than trying to bring the energy to an existing location.

The GreenStar Network (GSN) project [3] is one of the first worldwide initiatives aimed at providing ICT services based entirely on renewable energy sources such as solar, wind and hydroelectricity across Canada and around the world. The network can transport user service applications to be processed in data centers built in proximity to green energy sources, thus GHG emissions of ICT equipments are reduced to minimal. Whilst energy efficiency techniques are still encouraged at low-end client equipments (e.g., such as hand-held devices, home PCs), the heaviest computing services will be dedicated to data centers powered completely by green energy. This is enabled thanks to a large abundant reserve of natural green energy resources in Canada, Europe and USA. The carbon credit saving that we refer to in this paper is the emission due to the operation of the network; the GHG emission during the production phase of the equipments used in the network and in the server farms is not considered since no special equipment is deployed in the GSN.

In order to move virtualized data centers towards network nodes powered by green energy sources distributed in such a multi-domain network, particularly between Europe and North America domains, the GSN is based on a flexible routing platform provided by the Mantychore FP7 project [8], which collaborates with the GSN project to enhance the carbon footprint exchange standard for ICT services. This collaboration enables research on the feasibility of powering e-Infrastructures in multiple domains worldwide with renewable energy sources. Management and technical policies will be developed to leverage virtualization, which helps to migrate virtual infrastructure resources from one site to another based on power availability. This will facilitate use of renewable energy within the GSN providing an Infrastructure as a Service (IaaS) management tool. By integrating connectivity to parts of the European National Research and Education Network (NREN) infrastructures with the GSN network this develops competencies to understand how a set of green nodes (where each 
one is powered by a different renewable energy source) could be integrated into an everyday network. Energy considerations are taken before moving virtual services without suffering connectivity interruptions. The influence of physical location in that relocation is also addressed, such as weather prediction, estimation of solar power generation. Energy produced from renewable sources has the potential to become one of the industries in each of these countries. In addition to the GSN-Mantychore project, a number of other Green ICT projects are also conducted in parallel in the FP7 framework [16].

The main objective of the GSN/Mantychore liaison is to create a pilot and a testbed environment from which to derive best practices and guidelines to follow when building low carbon networks. Core nodes are linked by an underlying high speed optical network having up to $1,000 \mathrm{Gbit} / \mathrm{s}$ bandwidth capacity provided by CANARIE. Note that optical networks have a modest increase in power consumption, especially with new $100 \mathrm{Gbit} / \mathrm{s}$, in comparison to electronic equipments such as routers and aggregators [4]. The migration of virtual data centers over network nodes is indeed a result of a convergence of server and network virtualizations as virtual infrastructure management. The GSN as a network architecture is built with multiple layers, resulting in a large number of resources to be managed. Virtualized management has therefore been proposed for service delivery regardless of the physical location of the infrastructure which is determined by resource providers. This allows complex underlying services to remain hidden inside the infrastructure provider. Resources are allocated according to user requirements; hence high utilization and optimization levels can be achieved. During the service, the user monitors and controls resources as if he was the owner, allowing the user to run their application in a virtual infrastructure powered by green energy sources.

\section{Provisioning of ICT Services over Mantychore FP7 and GSN with Renewable Energy}

In the European NREN community connectivity services are provisioned on a manual basis with some effort now focusing towards automating the service setup and operation. Rising energy costs, working in an austerity based environment which has dynamically changing business requirements has raised the focus of the community to control some characteristics of these connectivity services, so that users can change some of the service characteristics without having to renegotiate with the service provider.

The Mantychore FP7 project has evolved from previous research projects MANTICORE and MANTICORE II [8][9]. The initial MANTICORE project goal was to implement a proof of concept based on the idea that routers and an IP network can be setup as a Service (IPNaaS, as a management Layer 3 network). MANTICORE II continued in the steps of its predecessor to implement stable and robust software while running trials on a range of network equipment.

The Mantychore FP7 project allows the NRENs to provide a complete, flexible network service that offers research communities the ability to create an IP network under their control, where they can configure: 
a) Layer 1, Optical links. Users will be able to get access control over optical devices like optical switches, to configure important properties of its cards and ports. Mantychore integrates the Argia framework [10] which provides complete control of optical resources.

b) Layer 2, Ethernet and MPLS. Users will be able to get control over Ethernet and MPLS (Layer 2.5) switches to configure different services. In this aspect, Mantychore will integrate the Ether project [6] and its capabilities for the management of Ethernet and MPLS resources.

c) Layer 3, Mantychore FP7 suite includes set of features for: i)Configuration and creation of virtual networks, ii) Configuration of physical interfaces, iii) Support of routing protocols, both internal (RIP, OSPF) and external (BGP), iv) Support of QoS and firewall services, v) Creation, modification and deletion of resources (interfaces, routers) both physical and logical, and vi) Support of IPv6. It allows the configuration of IPv6 in interfaces, routing protocols, networks.

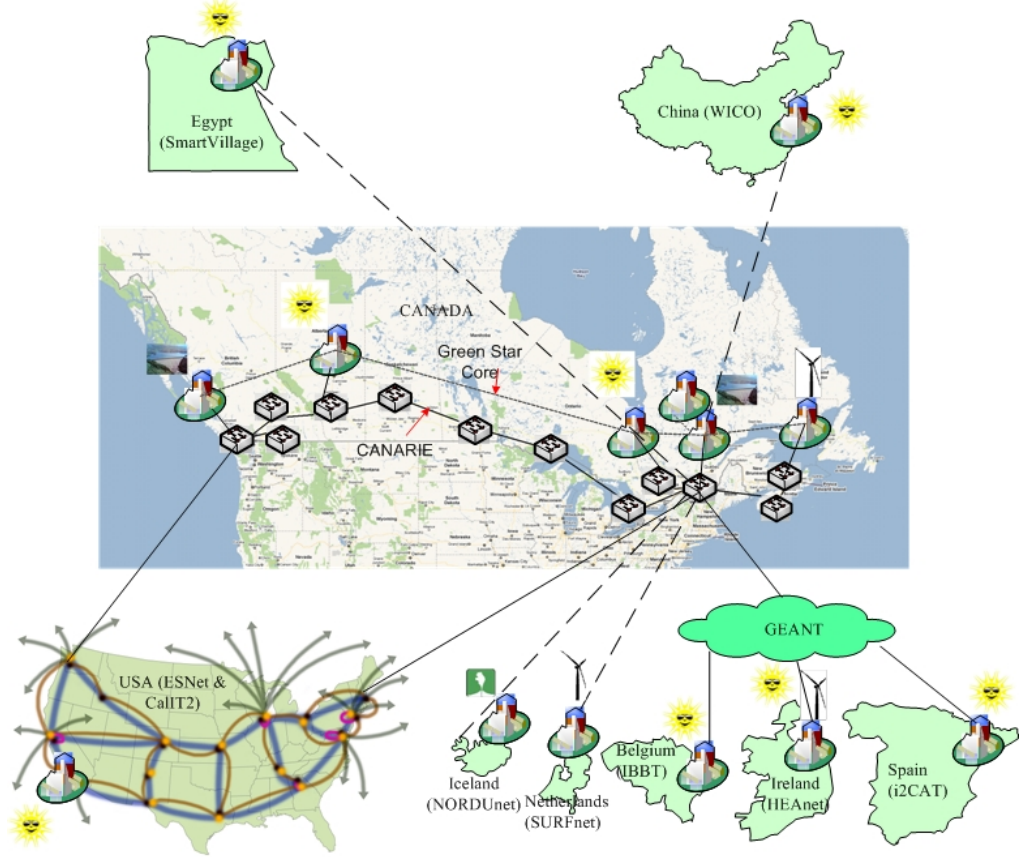

Fig. 1. The GreenStar Nework

Figure 1 shows the connection plan of the GSN. The Canadian section of the GSN has the largest deployment of six GSN nodes powered by sun, wind and hydroelectricity. It is connected to the European green nodes in Ireland (HEAnet), Iceland (NORDUnet), Spain (i2CAT), Belgium (IBBT), the Netherlands (SURFnet), and some other nodes in other parts of the world such as in China (WiCo), Egypt (Smart Village) and USA (ESNet).

One of the key objectives of the liaison between Mantychore FP7 and GSN projects is to enable renewable energy provisioning for NRENs. Building competency 
using renewable energy resources is vital for any NREN with such an abundance of natural power generation resources at their backdoor and has been targeted as a potential major industry for the future. HEAnet in Ireland [11], where the share of electricity generated from renewable energy sources in 2009 was $14.4 \%$, have connected two GSN nodes via the GEANT Plus service and its own NREN network to a GSN solar powered node in the South East of Ireland and to a wind powered grid supplied location in the North East of the country. NORDUnet [12], which links Scandinavian countries having the highest proportion of renewable energy sources in Europe, houses a GSN Node at a data center in Reykjavík (Iceland) and also contributes to the GSN/Mantychore controller interface development. In Spain [13], where $12.5 \%$ of energy comes from renewable energy sources (mostly solar and wind), i2CAT is leading the Mantychore development as well as actively defining the interface for GSN/Mantychore and they will setup a solar powered node in Lleida (Spain). There will be also a GSN node provided by the Interdisciplinary institute for BroadBand Technology (IBBT) network in Belgium [15]. IBBT is involved in several research projects on controlling energy usage and setting up dynamic architectures and reducing $\mathrm{CO} 2$ emission and they will setup a solar powered node in Gent in order to perform power related experiments.

\section{Architecture of a Zero-Carbon Network}

We now focus on the design and management of a GSN network which provides zero-carbon ICT services. The only difference between the GSN and a regular network is that the former one is able to transport ICT services to data centers powered

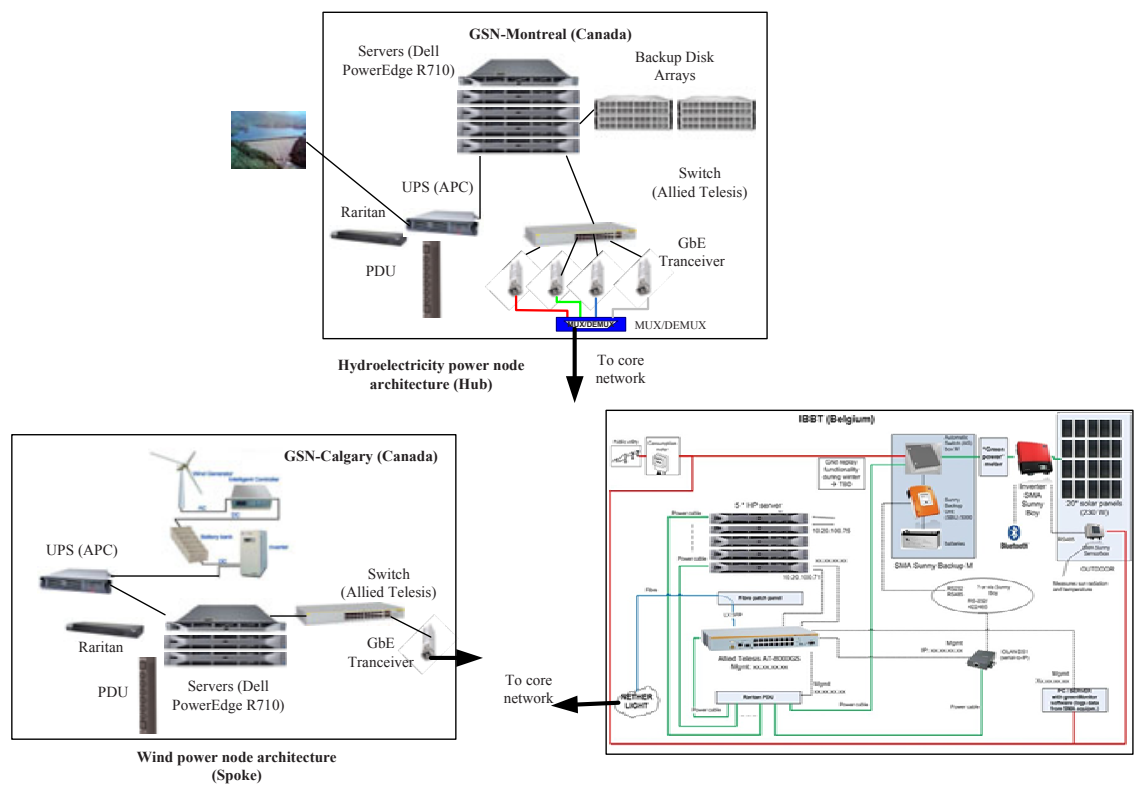

Fig. 2. Architecture of green nodes (hydro, wind and solar types) 
by green energy and adjust the network to the needs controlled by software. The cost of producing and maintaining network elements, such as routers and servers, is not considered, because no special hardware equipment is used in the GSN.

Figure 2 illustrates the architectures of a hydroelectricity and two green nodes, one is powered by solar energy and the other is powered by wind. The solar panels are grouped in bundles of 9 or 10 panels, each panel generates a power of $220-230 \mathrm{~W}$. The wind turbine system is a $15 \mathrm{~kW}$ generator. After being accumulated in a battery bank, electrical energy is treated by an inverter/charger in order to produce an appropriate output current for computing and networking devices. User applications are running on multiple Dell PowerEdge R710 systems, hosted by a rack mount structure in an outdoor climate-controlled enclosure. The air conditioning and heating elements are powered by green energy at solar and wind nodes; they are connected to regular the power grid at hydro nodes. The PDUs (Power Distribution Unit), provided with power monitoring features, measures electrical current and voltage. Within each node, servers are linked by a local network, which is then connected to the core network through GE transceivers. Data flows are transferred among GSN nodes over dedicated circuits (like light paths or P2P links), tunnels over Internet or logical IP networks.

The Montreal GSN node plays a role of a manager (hub node) that opportunistically sets up required connectivity for Layer 1 and Layer 2 using dynamic services, then pushes Virtual Machines (VMs) or software virtual routers from the hub to a sun or wind node (spoke node) when power is available. VMs will be pulled back to the hub node when power dwindles. In such a case, the spoke node may switch over to grid energy for running other services if it is required. However, GSN services are powered entirely by green energy. The VMs are used to run user applications, particularly heavy-computing services. Based on this testbed network, experiments and research are performed targeting cloud management algorithms and optimization of the intermittently-available renewable energy sources.

The cloud management solution developed in order to run the GSN enables the control of a large number of devices of different layers. With power monitoring and control capabilities, the proposed solution aims at distributing user-oriented services

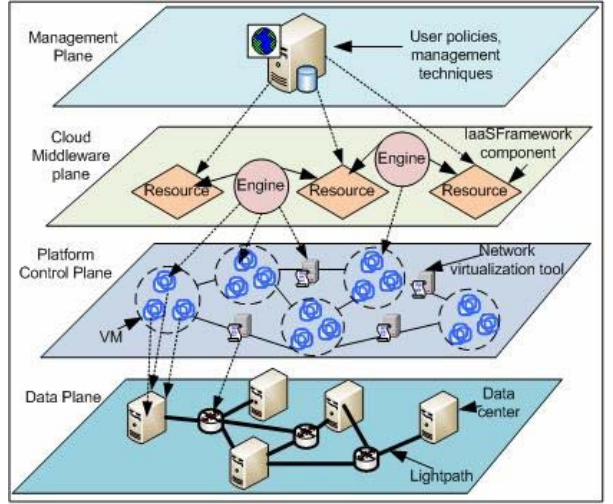

Layers of the GreenStar Network

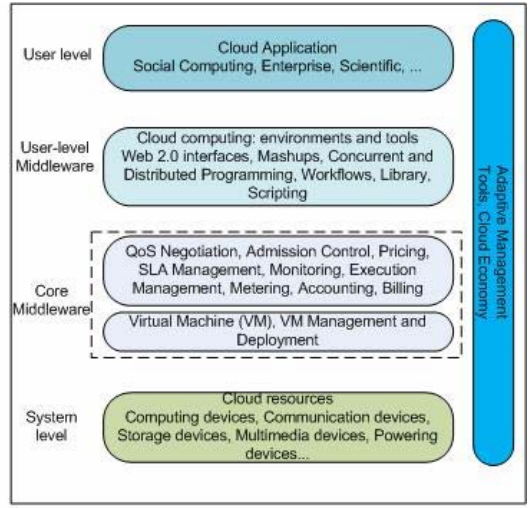

Reference layered model of a Cloud Architecture

Fig. 3. Layered GSN and Cloud Computing Architectures 
regardless of the underlying infrastructure. Such a management approach is essential for data center migration across a wide-area network, because the migration must be achieved in a timely manner and transparently to service users. The proposed webbased cloud management solution is based on the IaaS concept, which is a new software platform specific for dealing with the delivery of computing infrastructure [5].

Figure 3 compares the layered architecture of the GSN with a general architecture of a cloud comprising four layers. The GSN Data plane corresponds to the System level, including massive physical resources, such as storage servers and application servers linked by controlled circuits (i.e., lightpaths). The Platform Control plane corresponds to the Core Middleware layer, implementing the platform level services that provide running environment enabling cloud computing and networking capabilities to GSN services. The Cloud Middleware plane corresponds to the User-level Middleware, providing Platform as a Service capabilities based on IaaS Framework components [5]. The top Management plane or User level focuses on application services by making use of services provided by the lower layer services.

\section{$4 \quad$ Virtual Data Center Migration}

In the GSN project, we are interested in moving a virtual data center from one node to another. Such a migration is required for large-scale applications running on multiple servers with a high density connection local network. The migration involves four steps: i) Setting up a new environment (i.e., a new data center) for hosting the application with required configurations, ii) Configuring network connection, iii) Moving VMs and their running state information through this high speed connection to the new location, and iv) Turning off computing resources at the original node. Indeed, solutions for the migration of simple applications have been provided by many ICT operators in the market. However, large scale data centers require arbitrarily setting their complex working environments when being moved. This results in a reconfiguration of a large number of servers and network devices in a multi-domain environment.

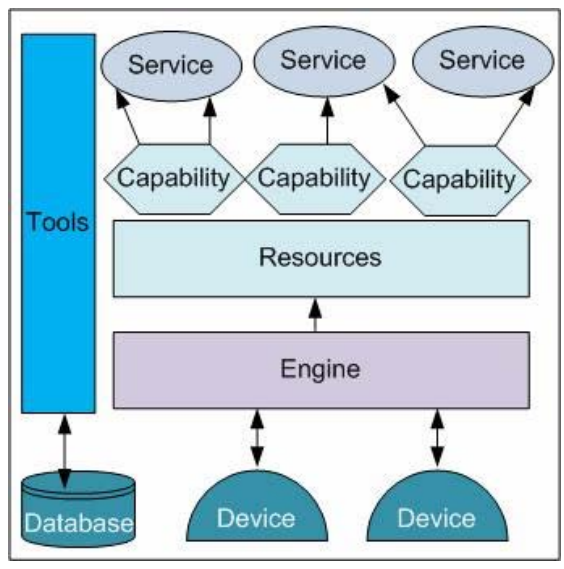

Fig. 4. IaaS Framework Architecture Overview 
In our experiments with an online interactive application like Geochronos [7] each VM migration requires $32 \mathrm{Mbps}$ bandwidth in order to keep the service live during the migration, thus a $10 \mathrm{Gbit} / \mathrm{s}$ link between two data centers can transport more than 300 VMs in parallel. Given that each VM occupies one processor and that each server has up to 16 processors, 20 servers can be moved in parallel. If each VM consumes 4GByte memory space, the time required for such a migration is $1000 \mathrm{sec}$.

The migration of data centers among GSN nodes is based on cloud management. The whole network is considered as a set of clouds of computing resources, which is managed using the IaaS Framework [5]. The IaaS Framework include four main components: i) IaaS Engine used to create model and devices interactions abstractions, ii) IaaS Resource used to build web services interfaces for manageable resources, iii) IaaS Service serves as a broker which controls and assigns tasks to each VM, and iv) IaaS Tool provides various tools and utilities that can be used by the three previous components (Figure 4).

The Engine component is positioned at the lowest level of the architecture and maintains interfaces with physical devices. It uses services provided by protocols and transport layers in order to achieve communications. Each engine has a state machine, which parses commands and decides to perform appropriate actions. The GSN management is achieved by three types of engines: i) Computing engine is responsible for managing VMs, ii) Power engine takes care of power monitoring and control and iii) Network engine controls network devices. The engines allow GSN users to quantify the power consumption of their service. Engines notify upper layers by triggering events. The Resource component serves as an intermediate layer between Engine and Service. It provides Service with different capabilities. Capabilities can contribute to a resources Business, Presentation or Data Access Tier. The Tool component provides additional services, such as persistence, which are shared by other components.

Based on the J2EE/OSGi platform, the IaaS Framework is designed in such a modular manner that each module can be used independently from others. OSGi (Open Services Gateway initiative) is a Java framework for remotely deployed service applications, which provides high reliability, collaboration, large scale distribution and wide-range of device usage. With an IaaS Framework based solution, the GSN can easily be extended to cover different layers and technologies.

Through a Web interface, users may determine GHG emission boundaries based on information providing VM power and their energy sources, and then take actions in order to reduce GHG emissions. The project is therefore ISO 14064 compliant. Indeed, cloud management has been addressed in many other research projects, e.g., [14], however, the IaaS Framework is chosen for the GSN because it is an open platform and converges server and network virtualizations. Whilst most of cloud management solutions in the market focus particularly on computing resources, IaaS Framework components can be used to build network virtualized tools [6][10], which provides for a flexible set of data flows among data centers. The ability of incorporating third-party power control components is also an advantage of the IaaS Framework. 


\section{$5 \quad$ Federated Network}

GSN takes advantage of the virtualization to link virtual resources together to span multiple cloud and substrate types. The key issue is how to describe, package, and deploy such multi-domain cloud applications. An orchestration middleware is built to federate clouds across domains, coordinate user registration, resource allocation, stitching, launch, monitoring, and adaptation for multi-domain cloud applications. Such a tool also requires solutions for identity, authorization, monitoring, and resource policy specification and enforcement. An extensible plug-in architecture has been developed in order to enable these solutions to evolve over time and leverage and interoperate with software outside of the GSN.

Along with the participation of international nodes, there is an increasing need of support for dynamic circuits on GSN, which is a multi-layer network, including interdomain links that span more than one network. Technologies to virtualize networks are continuing to advance beyond the VPN tunneling available to the early cloud network efforts. Some recently built multi-layer networks, like GENI, offer direct control of the network substrate to instantiate isolated virtual pipes, which may appear as VLANs, MPLS tunnels, or VPLS services at the network edge.

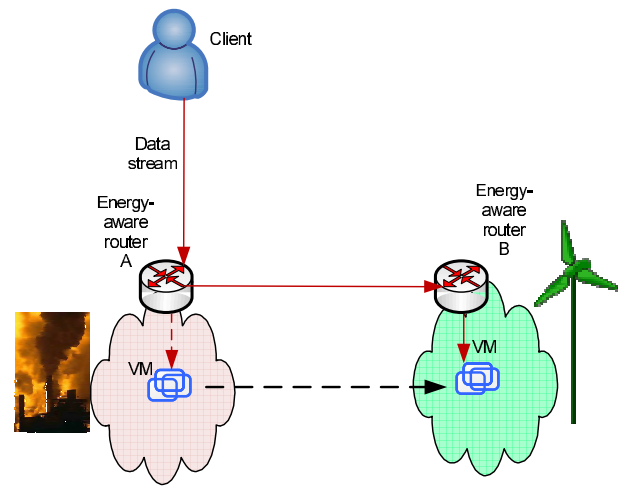

Fig. 5. Energy-aware routing

In the proposed new energy-ware routing scheme based on Mantychore support, the client will contact firstly an energy-aware router in order to get an appropriate VM for his service. The router will look for a VM which is optimal in terms of GHG emission, i.e., the one which is powered by a green energy source. If no VM is found, a migration process would be triggered in order to move a VM to a greener data center. The process is as follows (Figure 5): i) Copy VM memory between old and new locations, ii) VM sends an ARP, iii) Router $B$ receives the ARP and sends the message to the client, iv) New routing entry is installed in router B for the VM, and v) New routing entry is added in router $\mathrm{A}$. 
In our design, the GSN is provided with a component called the Federation Stitcher which is responsible for establishing connection among domains, and forwarding user requests to appropriate data centers. The big picture of the GSN network management solution is shown in Figure 6. The heart of the network is the programmable Federation Stitcher, which accepts connections from service users through Internet. This point is powered by green sustainable energy, i.e., hydroelectricity. It links user requests to appropriate services provided by data centers distributed across the network. Each data center is represented by a virtual instance, including virtual servers and virtual routers and/or virtual switches interconnecting the servers. Such a virtual data center can be hosted by any physical network node, according to the power availability. There is a domain controller within each data center or a set of data centers sharing the same network architecture/policy. User requests will be forwarded by the Federation Stitcher to the appropriate domain controller. When a VM or a data center is migrated, the new location will be registered with the Federation Stitcher then user requests are tunneled to the new domain controller.

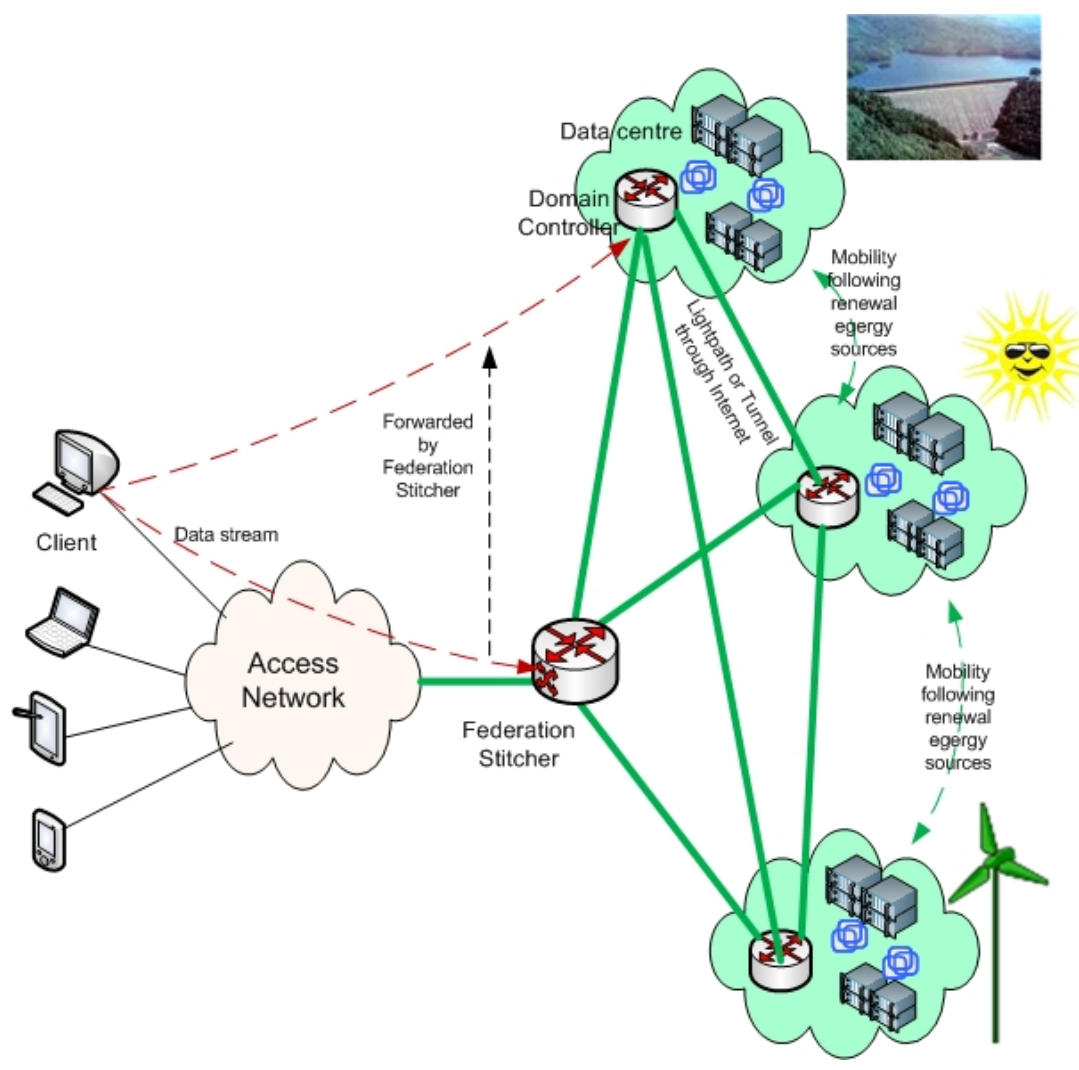

Fig. 6. Overview of GSN network management solution 


\section{Conclusion}

In this chapter, we have presented a prototype of a Future Internet powered only by green energy sources. As a result of the cooperation between Europe and North America researchers, the GreenStar Network is a promising model to deal with GHG reporting and carbon tax issues for large ICT organizations. Based on the Mantychore FP7 project, a number of techniques have been developed in order to provision renewable energy for ICT services worldwide. Virtualization techniques are shown to be the most appropriate solution to manage such a network and to migrate data centers following green energy source availability, such as solar and wind.

Our future work includes research on the quality of services hosted by the GSN and a scalable resource management.

Acknowledgments. The authors thank all partners for their contribution in the GSN and Mantychore FP7 projects.

Open Access. This article is distributed under the terms of the Creative Commons Attribution Noncommercial License which permits any noncommercial use, distribution, and reproduction in any medium, provided the original author(s) and source are credited.

\section{References}

1. The Climate Group: SMART2020: Enabling the low carbon economy in the information age. Report on behalf of the Global eSustainability Initiative, GeSI (2008)

2. Saunders, H.: The Khazzoom-Brookes Postulate and Neoclassical Growth. The Energy J 13(4) (1992)

3. The GreenStar Network Project, http: / /www.greenstarnetwork.com/

4. Figuerola, S., Lemay, M., Reijs, V., Savoie, M., St. Arnaud, B.: Converged Optical Network Infrastructures in Support of Future Internet and Grid Services Using IaaS to Reduce GHG Emissions. J. of Lightwave Technology 27(12) (2009)

5. Lemay, M.: An Introduction to IaaS Framework. (8/2008), http: / /www. iaasframework. com/

6. Figuerola, S., Lemay, M.: Infrastructure Services for Optical Networks. J. of Optical Communications and Networking 1(2) (2009)

7. Kiddle, C.: GeoChronos: A Platform for Earth Observation Scientists. OpenGridForum 28, $(3 / 2010)$

8. Grasa, E., Hesselbach, X., Figuerola, S., Reijs, V., Wilson, D., Uzé, J.M., Fischer, L., de Miguel, T.: The MANTICORE Project: Providing users with a Logical IP Network Service. TERENA Networking Conference (5/2008)

9. Grasa, E., et al.: MANTICORE II: IP Network as a Service Pilots at HEAnet, NORDUnet and RedIRIS. TERENA Networking Conference (6/2010)

10. Grasa, E., Figuerola, S., Forns, A., Junyent, G., Mambretti, J.: Extending the Argia software with a dynamic optical multicast service to support high performance digital media. Optical Switching and Networking 6(2) (2009)

11. HEAnet website, http: //www. heanet. ie/

12. NORDUnet website, http: //www. nordu. net

13. Moth, J.: GN3 Study of Environmental Impact Inventory of Greenhouse Gas Emissions and Removals - NORDUnet (9/2010)

14. OpenNEbula Project, http: //www. opennebula .org/

15. IBBT Website, http: //www. ibbt.be/

16. Reservoir FP7, http: //www.reservoir-fp7.eu/ 